\title{
Asymptomatic primary tuberculous pleurisy with intense 18-fluorodeoxyglucose uptake mimicking malignant mesothelioma
}

\author{
Tsutomu Shinohara $^{1 *}$, Naoki Shiota ${ }^{2}$, Motohiko Kume $^{3}$, Norihiko Hamada ${ }^{4}$, Keishi Naruse ${ }^{5}$ and Fumitaka Ogushi ${ }^{6}$
}

\begin{abstract}
Background: The pathogenesis of primary tuberculous pleurisy is a delayed-type hypersensitivity immunogenic reaction to a few mycobacterial antigens entering the pleural space rather than direct tissue destruction by mycobacterial proliferation. Although it has been shown that pulmonary tuberculosis induces 18-fluorodeoxyglucose (FDG) uptake in active lesions, little is known about the application of FDG positron emission/computed tomography (FDG PET/CT) to the management of primary tuberculous pleurisy.
\end{abstract}

Case presentation: We report a case of asymptomatic primary tuberculous pleurisy presenting with diffuse nodular pleural thickening without distinct pleural effusion and parenchymal lung lesions mimicking malignant mesothelioma. An initial FDG PET/CT scan demonstrated multiple lesions of intense FDG uptake in the right pleura and thoracoscopic biopsy of pleural tissue revealed caseous granulomatous inflammation. The patient received antituberculous therapy for 6 months, with clearly decreased positive signals on a repeated FDG PET/CT scan.

Conclusion: FDG PET/CT imaging may be useful for evaluating disease activity in tuberculous pleurisy patients with an unknown time of onset.

Keywords: Primary tuberculous pleurisy, Fluorodeoxyglucose, Positron emission tomography

\section{Background}

18-fluorodeoxyglucose positron emission/computed tomography (FDG PET/CT) is a noninvasive imaging technique that visualizes glucose metabolism and has been regarded as useful for differentiating between benign and malignant diseases [1,2]. However, it has been shown that inflammatory disorders, especially pulmonary tuberculosis, also induce FDG uptake in active lesions, which suggests that FDG PET results should be interpreted with caution [3-5]. Conventional or dual phase FDG PET can not effectively distinguish malignant disease from mycobacterium infections. Multi-tracer PET, a novel promising technology utilizing differences in tracer kinetics and decay, has been providing additional and complementary information to improve pulmonary nodule characterization [5].

The usefulness of FDG PET in the detection of mycobacterium infection sites has been shown in patients with

\footnotetext{
* Correspondence: shinoharat@kochi2.hosp.go.jp

'Department of Clinical Investigation, National Hospital Organization National Kochi Hospital, 1-2-25 Asakuranishimachi, Kochi 780-8077, Japan

Full list of author information is available at the end of the article
}

miscellaneous infectious lesions or human immunodeficiency virus-associated fever of unknown origin [6,7]. Moreover, it has been reported that FDG PET/CT is helpful not only for evaluating disease activity, but also for monitoring therapeutic responses in pulmonary tuberculosis, tuberculous lymphadenitis, and skeletal tuberculosis, particularly in smear-negative patients [5,8-10]. Via et al. demonstrated that FDG-PET uptake in tuberculous pulmonary lesions was significantly reduced with as little as 1 week of treatment prior to changes in the volume and density of lesions in a rabbit model [11]. The absence of reduced FDG PET uptake in the early phase of treatment may suggest active tuberculosis due to a lack of adherence, drug resistance, or misdiagnosis [9].

The pathogenesis of primary tuberculous pleurisy is a delayed-type hypersensitivity immunogenic reaction to a few mycobacterial antigens entering the pleural space rather than direct tissue destruction by mycobacterial proliferation [12]. However, little is known about the application of FDG PET/CT imaging to the management of primary tuberculous pleurisy. We report here a case 

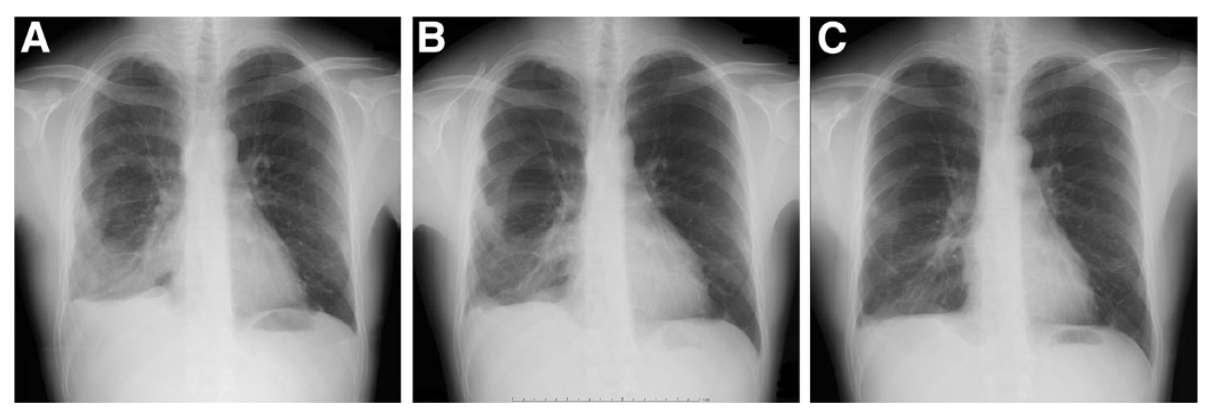

Figure 1 Chest radiograph. A: Pleural based opacities in the right hemithorax at presentation. B: Marked improvements after 6 months of antituberculous chemotherapy. C: Further improvements another 6 months after the completion of chemotherapy.

of asymptomatic primary tuberculous pleurisy with intense FDG uptake mimicking malignant mesothelioma.

\section{Case presentation}

A 26-year-old man with no subjective symptoms was referred to our hospital because of a chest radiographic abnormality in the right hemithorax on his chest X-ray (Figure 1A), which was incidentally found during a college health examination. Chest CT revealed diffuse nodular thickening of the right pleura, without distinct pleural effusion and parenchymal lung lesions, mimicking malignant mesothelioma. An initial FDG PET/CT scan demonstrated multiple lesions of intense FDG uptake in the right pleura (maximal standard uptake value $(S U V \max )=10.9)$ (Figure 2A and Figure 3A). He had no history of pulmonary or autoimmune disease or occupational exposure to asbestos, silica, or other fibrogenic substances. Since the pleural specimens obtained from CT-guided needle biopsy were insufficient to allow for a definitive diagnosis, the patient was admitted for thoracoscopic biopsy.

On admission, right breath sounds were decreased. Remaining physical examinations revealed no abnormal findings. A full blood cell count, the results of liver and renal function tests, and other data were within normal limits. Thoracoscopic biopsy of the pleural tissue revealed caseous necrosis and granulomatous inflammation (Figure 4), although acid-fast bacilli were not found in biopsy specimens. Cultures of sputum and pleural tissue were also negative. However, the QuantiFERON-TB second generation $(\mathrm{QFT}-2 \mathrm{G})$ test $(3.02 \mathrm{IU} / \mathrm{ml})$ and tuberculin skin test (10 $\mathrm{mm}$ induration) were both positive based on the Japanese criteria.

Although no positive culture was obtained, other clinical data suggested that a diagnosis of tuberculous pleurisy was highly likely in this case and the patient received antituberculous therapy for 6 months (2HREZ/4HRE) without any side effects. Just after chemotherapy, a repeated FDG PET/CT scan revealed clearly decreased positive signals (SUVmax $=5.7)$ with marked improvements in pleural thickening (Figure 2B and Figure 3B). Six months after the completion of chemotherapy, a chest radiograph showed further improvements in pleural based opacities relative to that taken just after chemotherapy (Figure $1 \mathrm{~B}$ and $\mathrm{C}$ ).
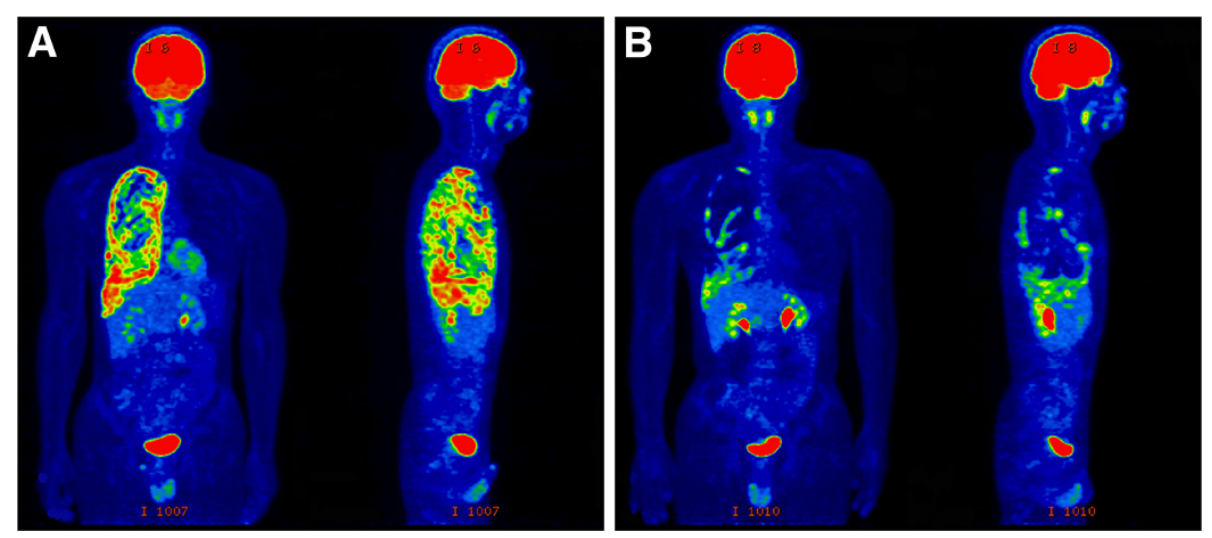

Figure 2 FDG PET image. A: Multiple lesions of intense FDG uptake in the right pleura at presentation. B: Clearly decreased positive signals after 6 months of antituberculous chemotherapy. 

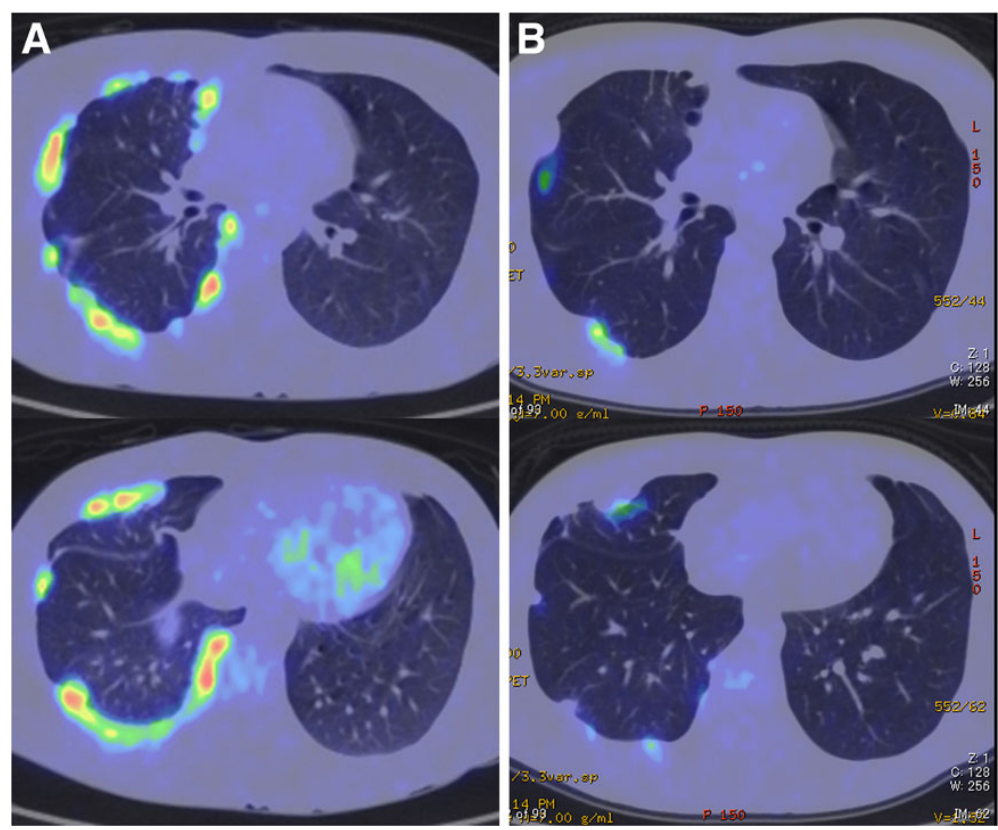

Figure 3 FDG PET/CT scan. A: Extensive nodular thickening of the right pleura at presentation showing intense FDG uptake without distinct pleural effusion and parenchymal lung lesions. B: Marked improvements with decreased positive signals after 6 months of antituberculous chemotherapy.

\section{Discussion}

Pleural inflammation caused by bacteria, mycobacterium, or autoimmune disease is usually characterized by effusion, fever, malaise, chest pain, nonproductive cough, and dyspnea in the acute phage [13], or diffuse thickening of the pleura, with or without calcification, in the chronic phase. Therefore, pleural thickening with no subjective symptoms generally implies non-active inflammatory pleural diseases, so-called "residual pleural thickness", or neoplasm of the pleura. Interestingly, the asymptomatic diffuse pleural thickening in our case showed intense FDG uptake and proved to be caseous granulomatous inflammation.

Duysinx et al. suggested that FDG PET/CT is an effective tool for differentiating between benign and malignant pleural diseases. The uptake of FDG was intense in all cases of mesothelioma and neoplastic pleurisy associated with primary lung cancer. In contrast, FDG PET/CT imaging revealed an absence of FDG uptake within the pleura of 2 of 3 patients with tuberculous pleurisy and the rest showed moderate uptake, although detailed clinical data of these patients were not described [14]. Kramer et al. also evaluated the feasibility of PET in the diagnosis of pleural thickening on CT. They concluded that qualitative assessment with PET discriminates between malignant and benign pleural thickening with high accuracy and a high negative predictive value. However, there has been no description of tuberculous pleurisy [15]. To the best of our knowledge, this is the first reported case of tuberculous pleurisy with intense FDP uptake.

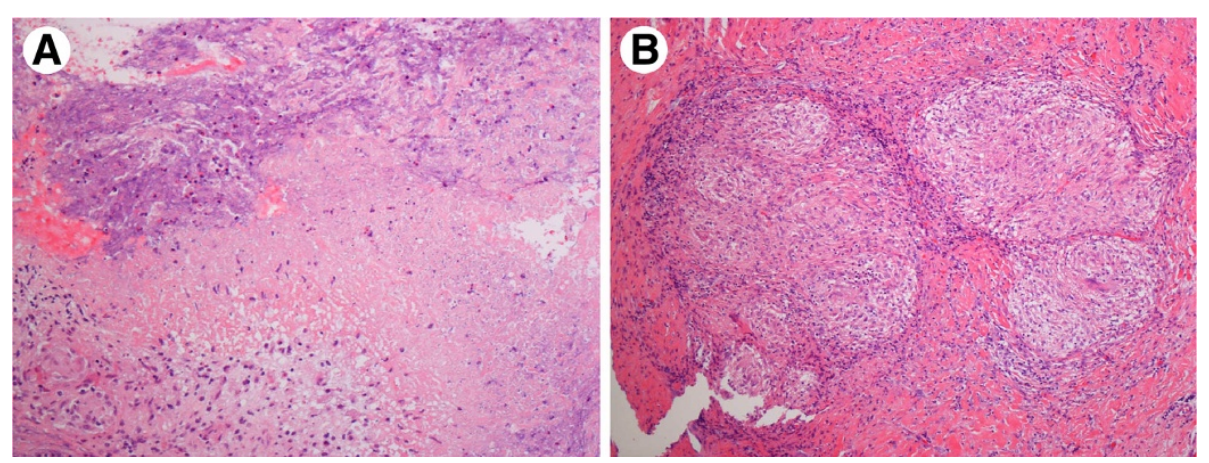

Figure 4 Pathological findings of pleural tissue. A: Caseous necrosis. B: Granulomatous inflammation. 
The pathogenesis of primary tuberculous pleurisy is a delayed-type hypersensitivity immunogenic reaction to a few mycobacterial antigens entering the pleural space rather than direct tissue destruction by uncontrolled mycobacterial proliferation [12]. This appears to be the reason for the low rate of positive results in acid-fast staining of pleural fluid and tissue, as seen in this case [16]. Recent findings suggest that the immunogenic reaction in tuberculous pleurisy involves lymphocytes (including CD3, CD4, CCR4, and Th17 cells), neutrophils, mesothelial cells, and various cytokines such as interferon- $\gamma$, interleukin (IL)-8, IL-12, and monocyte chemoattractant protein-1 $[17,18]$. However, the complex mechanisms that induce high glucose metabolism and show high uptake of FDG in pleural thickening is unclear at this time.

About half of untreated cases of primary tuberculous pleurisy relapse with more severe forms of active pulmonary or extra-pulmonary tuberculosis, which may result in severe disability or death $[19,20]$. However, in general, many cases of pleural thickening suspiciously caused by primary tuberculous pleurisy with no subjective symptoms have not received antituberculous chemotherapy. FDG uptake in the pleura with positive QuantiFERON-TB may support the use of antituberculous treatment in such cases.

\section{Conclusions}

Active tuberculosis should be considered in the differential diagnosis of diffuse pleural thickening even when there are no subjective symptoms. Moreover, FDG PET/ CT imaging may be useful for evaluating disease activity and monitoring therapeutic responses in tuberculous pleurisy patients with an unknown time of onset.

\section{Consent}

Written informed consent was obtained from the patient for publication of this case report and any accompanying images. A copy of the written consent is available for review by the Editor-in-Chief of this journal.

\section{Abbreviations \\ FDG: 18-fluorodeoxyglucose; PET: Positron emission tomography; CT: Computed tomography; SUVmax: Maximal standard uptake value; QFT- 2G: QuantiFERON-TB second generation; IL: Interleukin.}

\section{Competing interests}

The authors declare that they have no competing interests.

\section{Authors' contributions}

TS was directly involved in the diagnosis and treatment of the patient, manuscript preparation, editing, and submission. NS contributed to patient care and collected patient data. MK performed the surgical procedure. KN performed pathologic studies. $\mathrm{NH}$ was the reference radiologist during the management of this case. FO was the attending physician throughout the disease course. All authors read and approved the final manuscript.

\section{Author details}

'Department of Clinical Investigation, National Hospital Organization National Kochi Hospital, 1-2-25 Asakuranishimachi, Kochi 780-8077, Japan.
${ }^{2}$ Department of Hematology and Respiratory Medicine, Kochi Medical School, Kochi University, Nankoku, Kochi 783-8505, Japan. ${ }^{3}$ Department of Surgery II, Kochi Medical School, Kochi University, Nankoku, Kochi 783-8505, Japan. ${ }^{4}$ Department of Radiology, Kochi Medical School, Kochi University, Nankoku, Kochi 783-8505, Japan. ${ }^{5}$ Department of Pathology, Kochi National Hospital Organization National Kochi Hospital, 1-2-25 Asakuranishimachi, Kochi 780-8077, Japan. ${ }^{6}$ Division of Pulmonary Medicine, National Hospital Organization National Kochi Hospital, 1-2-25 Asakuranishimachi, Kochi 780-8077, Japan.

Received: 17 September 2012 Accepted: 11 January 2013

Published: 14 January 2013

\section{References}

1. Strauss LG: Positron emission tomography: current role for diagnosis and therapy monitoring in oncology. Oncologist 1997, 2:381-388.

2. Gould MK, Maclean CC, Kuschner WG, Rydzak CE, Owens DK: Accuracy of positron emission tomography for diagnosis of pulmonary nodules and mass lesions: a meta-analysis. JAMA 2001, 285:914-924.

3. Zheng Z, Pan Y, Guo F, Wei H, Wu S, Pan T, Li J: Multimodality FDG PET/CT appearance of pulmonary tuberculoma mimicking lung cancer and pathologic correlation in a tuberculosis-endemic country. South Med 2011, 104:440-445.

4. Soussan M, Brillet PY, Mekinian A, Khafagy A, Nicolas P, Vessieres A, Brauner M: Patterns of pulmonary tuberculosis on FDG-PET/CT. Eur J Radiol 2012, 81:2872-2876.

5. Treglia G, Taralli S, Calcagni ML, Maggi F, Giordano A, Bonomo L: Is there a role for fluorine 18 fluorodeoxyglucose-positron emission tomography and positron emission tomography/computed tomography in evaluating patients with mycobacteriosis? A systematic review. J Comput Assist Tomogr 2011, 35:387-393.

6. Ichiya Y, Kuwabara Y, Sasaki M, Yoshida T, Akashi Y, Murayama S, Nakamura K, Fukumura T, Masuda K: FDG-PET in infectious lesions: The detection and assessment of lesion activity. Ann Nucl Med 1996, 10:185-191.

7. Castaigne C, Tondeur M, de Wit S, Hildebrand M, Clumeck N, Dusart M: Clinical value of FDG-PET/CT for the diagnosis of human immunodeficiency virus-associated fever of unknown origin: a retrospective study. Nucl Med Commun 2009, 30:41-47.

8. Park IN, Ryu JS, Shim TS: Evaluation of therapeutic response of tuberculoma using F-18 FDG positron emission tomography. Clin NuCl Med 2008, 33:1-3.

9. Martinez V, Castilla-Lievre MA, Guillet-Caruba C, Grenier G, Fior R, Desarnaud S, Doucet-Populaire F, Boué F: ${ }^{18}$ F-FDG PET/CT in tuberculosis: an early non-invasive marker of therapeutic response. Int J Tuberc Lung Dis 2012, 16:1180-1185

10. Go SW, Lee HY, Lim CH, Jee WH, Wang YP, Yoo IR, Kang JY: Atypical disseminated skeletal tuberculosis mimicking metastasis on PET-CT and MRI. Intern Med 2012, 51:2961-2965.

11. Via LE, Schimel D, Weiner DM, Dartois V, Dayao E, Cai Y, Yoon YS, Dreher MR, Kastenmayer RJ, Laymon CM, Carny JE, Flynn JL, Herscovitch P, Barry CE 3rd: Infection dynamics and response to chemotherapy in a rabbit model of tuberculosis using $\left[{ }^{18} \mathrm{~F}\right] 2$-fluoro-deoxy-D-glucose positron emission tomography and computed tomography. Antimicrob Agents Chemother 2012, 56:4391-4402.

12. Barnes PF, Mistry SD, Cooper CL, Pirmez C, Rea TH, Modlin RL Compartmentalization of a CD4+ T lymphocyte subpopulation in tuberculous pleuritis. J Immunol 1989, 142:1114-1119.

13. Levine H, Szanto PB, Cugell DW: Tuberculous pleurisy. An acute illness. Arch Intern Med 1968, 122:329-332.

14. Duysinx B, Nguyen D, Louis R, Cataldo D, Belhocine T, Bartsch P, Bury TJ: Evaluation of pleural disease with 18-fluorodeoxyglucose positron emission tomography imaging. Chest 2004, 125:489-493.

15. Kramer H, Pieterman RM, Slebos DJ, Timens W, Vaalburg W, Koëter GH, Groen HJ: PET for the evaluation of pleural thickening observed on CT. J Nucl Med 2004, 45:995-998.

16. Gopi A, Madhavan SM, Sharma SK, Sahn SA: Diagnosis and treatment of tuberculous pleural effusion in 2006. Chest 2007, 131:880-889.

17. Ferrer Sancho J: Pleural tuberculosis: incidence, pathogenesis, diagnosis, and treatment. Curr Opin Pulm Med 1996, 2:327-334.

18. Caramori G, Lasagna L, Casalini AG, Adcock IM, Casolari P, Contoli M, Tafuro F, Padovani A, Chung KF, Barnes PJ, Papi A, Rindi G, Bertorelli G: Immune 
response to Mycobacterium tuberculosis infection in the parietal pleura of patients with tuberculous pleurisy. PLoS One 2011, 6:e22637.

19. Patiala J: Initial tuberculous pleuritis in the Finnish armed forces in 1939-1945 with special reference to eventual postpleuritic tuberculosis. Acta Tuberc Scand 1954, 36(Suppl):1-57.

20. Roper $W H$, Waring JJ: Primary serofibrinous pleural effusion in military personnel. Am Rev Tuberc 1955, 71:616-634.

doi:10.1186/1471-2334-13-12

Cite this article as: Shinohara et al:: Asymptomatic primary tuberculous pleurisy with intense 18-fluorodeoxyglucose uptake mimicking malignant mesothelioma. BMC Infectious Diseases 2013 13:12.

\section{Submit your next manuscript to BioMed Central and take full advantage of:}

- Convenient online submission

- Thorough peer review

- No space constraints or color figure charges

- Immediate publication on acceptance

- Inclusion in PubMed, CAS, Scopus and Google Scholar

- Research which is freely available for redistribution 